\title{
IMPLICATIONS OF THE $\tau$ AND b SPINS FOR THEORIES OF QUARKS AND LEPTONS
}

\author{
G.L. KANE \\ Physics Department, University of Michıgan, Ann Arbor, MI 48109, USA \\ and
}

\author{
S. RABY \\ Physics Department, Stanford University, Stanford, CA 94305, USA
}

Received 12 August 1979

\begin{abstract}
We argue that the present evidence that the spin of the $\tau 1$ s $1 / 2$ s circumstantial, and that a spin of $3 / 2$ is not excluded. If the $\tau$ spin were $3 / 2$, it would have important implications for the idea that leptons and quarks were composite states, perhaps allowing an explanation of the existence of three generations Supergravity ideas could also be affected. Similar ideas suggest the b-quark could have spm- $3 / 2$; we give several ways to test this.
\end{abstract}

Introduction. We have considered in some detail the possibility of constructing and of testing theories in which leptons and quarks are composite states of some other (presumably "more fundamental") objects; such a point of view has increasingly been studied $[1-5]$.

There are a number of reasons why this can be an attractive idea. It could relate leptons and quarks and account for the relation between lepton and quark charges (charge quantization). It could explain the existence of three, apparently equivalent, generations - the old $\mu$-e problem. It would reduce the number of fundamental fermions by a large factor, perhaps leading to a set of particles which would form a simple representation under supergravity. It could lead to new approaches to fermion masses and quark mixing angles.

Alternatively, there are reasons why lepton and quark substructure might be unattractive. Perhaps the strongest reason is that it would be nice if the particles we are studying now were really to be the ultimate buildıng blocks of nature, and economy would appear not in the number of fundamental particles but in underlying principles which would account for them. More technically, there is some reason to believe that quarks and leptons are in multiplets showing real group structure, exhibitıng relationships beyond those just indicated by countıng.

If any of the tests we suggest below were found to be positive it would make the possibility of quark and lepton substructure a much more serious one. Converse ly, if negative it would become more difficult to believe the present particles were not fundamental.

So far most proposals for quark and lepton substructure have been beyond reproach because there was no way to test them or argue against them. The theories effectively split into two classes, one where the dynamics is likely to be qualitatively consistent with present understanding of relativistic field theories, and one where the dynamics would have to be considered as mysterious at present. We argue that in the former class it is probably required that the spins of the $\tau$ and the b-quark (and $\nu_{\tau}$ and t-quark) be $3 / 2$, and there are a number of ways to test this. In the latter class, of course, tests will not be possible at present and one is free to speculate.

Before we discuss composite leptons and quarks, we treat the $\tau$ spin question since that is of considerable interest regardless of the validity of our comments about the leptons and quarks.

The $\tau$ spin. It has been argued and accepted that the $\tau$ spin is $1 / 2$ [6-8]. We do not argue that it is not $1 / 2$. We agree that all $\tau$ behavior is consistent with $S_{\tau}$ $=1 / 2$. However, we claim that there is no direct test, and that much or all of the observed behavior might 
change little if $S_{\tau}=3 / 2 \neq 1 \quad$ Further, the stakes are large - it would have dramatic implications for particle physics if $S_{\tau}=3 / 2$.

The usual argument for $S_{\tau}=1 / 2$, due to Tsai [6], appears to be reasonable, namely the cross section $\sigma\left(\mathrm{e}^{+} \mathrm{e}^{-} \rightarrow \tau^{+} \tau^{-}\right)$calculated from the usual photon contribution would grow faster than experument by at least a power of the center-of-mass energy squared, $s$. However, it is not conclusive for the following reason. The extra growth with energy arises from the longitudinal (helicity-1/2) degrees of freedom of the massive spin-3/2 particle; these are quite analogous to the longitudinal polarization states of a massive vector boson in a spontaneously broken gauge theory. In the latter case the growing amplitudes are cancelled by contributions from other particles in tree diagrams [10].

If the $\tau$ had $S_{\tau}=3 / 2$ as a consequence of a fundamental theory, it is certainly reasonable to expect that the theory would be unitary or renormalizable and that the $\mathrm{e}^{+} \mathrm{e}^{-} \rightarrow \tau^{+} \tau^{-}$amplitudes would not grow with energy when the full theory were included. It is possible that the damping would only occur as a form factor effect. Then to make sense it must occur only for very high energies since we already know [11] that no structure is present for e, $\mu$ at $s \lesssim 1000 \mathrm{GeV}^{2}$ (see below for further discussion of this). In this case perhaps only the usual photon would contribute at present energies and Tsai's argument would apply.

Alternatively, it could happen that the $\tau$ and $b$ had spin-3/2 and for reasons we would not understand without a fundamental theory, their interactions were described by an (effectively) renormalizable theory at all energies. Then it is entirely possible that all present data are consistent with $S_{\tau}=3 / 2$.

Ideally, we would write a consistent theory with a massive spin-3/2 particle coupled to the electromagnetıc current and containing spin- $1 / 2$ states. In that theory we could calculate and give an explicit example with $S_{\tau}=3 / 2$ and agreement with experiment for $R_{\tau}$ and $\tau$ decays, or fall to get agreement Unfortunately it is not easy to do that at the present time. Using insights from supergravity theories [12], which contain spin- $3 / 2$ particles, we have made some effort to

\#1 The possibility that $S_{\tau}=3 / 2$ has also been considered by the authors in ref [9] These authors have given forms of couplings which can give different results for different spin assignments, but have not considered general couplings. find such a theory, and have not yet been successful. Hopefully, we could find a consistent low energy theory with explicit threshold cancellations to be used only in tree approximation.

It is easy to see that a spin-3/2 theory could be of a form so as to give agreement with present data. For example, to give a prescription which is only meant to be illustrative and not as a serious approach, suppose that the theory were such as to simply remove the growing part of the longitudinal contribution. Then

$$
\begin{aligned}
& R\left(\mathrm{e}^{+} \mathrm{e}^{-} \rightarrow \tau^{+} \tau^{-}\right) \propto(\sqrt{1 / 3})^{2}+(2 / 3)^{2}\left(\epsilon^{0}\left(\tau^{+}\right) \epsilon^{0}\left(\tau^{-}\right)\right)^{2} \\
& \quad \rightarrow 1 / 3+4 / 9=7 / 9,
\end{aligned}
$$

which is not inconsistent with present data [13]. The first piece comes from producing one $\tau$ with helicity$3 / 2$ and the other with helicity- $1 / 2$, the second from one $\tau$ with helicity $-1 / 2$ and the other with helicity $-1 / 2$. With no cancellations $\epsilon^{0}\left(\tau^{+}\right) \epsilon^{0}\left(\tau^{-}\right)=s / 2 m_{\tau}^{2}-1$ and our illustrative prescription is simply to assume the $s / 2 m_{\tau}^{2}$ in this is cancelled by another contribution in the full theory. The numerical factors are the usual coefficients in the Rarita-Schwinger wavefunction. For decays one could simply couple $\left(\tau, \nu_{\tau}\right)$ as a SU(2) $X \mathrm{U}(1)$ doublet to a conventional $\mathrm{W}^{ \pm}$boson. Then all other particles would come from the other end of the $\mathrm{W}^{ \pm}$as in a conventional view. All that could change would be the ratio of longitudinal to transverse W's, which could change the ratio $\Gamma(\tau \rightarrow \pi \nu) / \Gamma(\tau \rightarrow \ell \nu \bar{\nu})$.

We can say a little more. Since the growing parts of the usual photon contribution to $R$, from $\epsilon^{0}\left(\tau^{+}\right) \epsilon^{0}\left(\tau^{-}\right) / s$, would have to be cancelled both at low energies where data is, and at high energies, cancellation by a massive $s$-channel amplitude $\left(1 /\left(m^{2}-s\right)\right)$ would not do. However, there are other contributions which can arrange 1t. First, supergravity theories have contact terms, and one with the form $\bar{\tau} \gamma_{\alpha} \tau \overline{\mathrm{e}} \gamma_{\alpha}$ e has a contribution which can cancel exactly the growing part of the photon amplitudes at all energies Second, $t$-channel contributions $\left(1 /\left(m^{2}-t\right)\right)$ can cancel at all energies, perhaps in different amounts at low and high energies. Finally, a massive spin-2 gauge particle $s$-channel contribution can have its pole removed by the propagator polynomals and give a piece which can cancel the photon part identically,

$Q_{\mu}\left(g_{\mu \nu}-Q_{\mu} Q_{\nu} / M^{2}\right) /\left(M^{2}-Q^{2}\right)=Q_{\nu} / M^{2}$.

Of course, all these contributions may give other terms 
with growing powers, but it does not seem unreasonable at this stage to hope that a full theory can be constructed where all divergences cancel. Also, it has been shown [14] for spin-0-spin-1/2 scattering in $N=2$ supergravity that the photon and graviton amplitudes can have a precise cancellation at threshold, although additional contributions would be required to give cancellations at high energies.

Since it is not known how to write a practical, meaningful, theory for massive spin-3/2 at the present time, the best way to proceed to test the $\tau$ spin is to tighten the tests of spin-1/2, which are more unique (technically they are not, but if $S_{\tau}=1 / 2$ there is no reason to embed it in a more complicated theory). In particular, measuring $R$ and the production angular distribution (checking the $\sin ^{2} \theta / s$ term) as well as possible would be worthwhile.

Composite quarks and leptons. Next we discuss the logic and value of having composite quarks and leptons, which leads to interestıng tests for the possibility that $S_{\mathrm{b}}=3 / 2$.

If considering quarks and leptons as composite could explain the existence of the e, $\mu$, and $\tau$ generations, unify quarks and leptons, and reduce the number of fundamental fermions, it would be an attractive idea. Alternatively, if it falled to do these it might seem considerably less attractive.

It is known [11] that $\mathrm{e}, \mu$ show no structure down to distances of order $10^{-16} \mathrm{~cm}$, or on mass scales of order $100 \mathrm{GeV}$. On this scale e, $\mu, \tau$ (and associated quarks, neutrinos) have essentially degenerate masses. Therefore if e, $\mu, \tau$ are to be explained it will not be as different radial or orbital states in some dynamical system, because these would be separated in energy by $\Delta E \approx 100 \mathrm{GeV}$.

The class of theories in which e, $\mu, \tau$ are constructed by taking three elementary fermions and adding spins might not suffer from this problem. The two spins give 0 or 1 , and the third spin gives two spin-1/2 particles (presumably e, $\mu$ ) and a spin-3/2 particle (presumably $\tau$ ). Choosing charges (e.g. as in ref. [5]) associates particles according to the usual content of grand unified representations $\left(\mathrm{e}, \mathrm{u}, \overline{\mathrm{d}}, \nu_{\mathrm{e}}\right.$ have spin-1/2 with one internal arrangement; $\mu, \mathrm{c}, \overline{\mathrm{s}}, \nu_{\mu}$ spin-1/2 with the other internal arrangement; and $\tau, \mathrm{t}, \mathrm{b}, \nu_{\tau}$ have spin$3 / 2)^{\ddagger 2}$. All other theories [15] appear etther not to explain the existence of three generations, or to require energy level structure on a scale small compared to the parameters of the theory. While this does not make them wrong automatically, it does put a burden of proof of relevance on them. In any case, these arguments also lead to the notion that $S_{\mathrm{b}}=3 / 2$.

Testing the b-quark spin. There are a number of tests of $S_{\mathrm{b}}$. Most dramatic, if $S_{\mathrm{b}}=3 / 2$ and $S_{\mathrm{u}, \mathrm{d}}=1 / 2$ the lowest-lying mesons in the B system stable against strong and electromagnetic decays will be spın-1, not spin -0 as for charm. The $\Upsilon$ spectrum will have four s-states, with spins 0,1,2,3. QCD-based predictions for the bound state spectrum and radiative decays when spin-3/2 fermions are involved could well be different, though they might not be.

The $b$ decays could be very different. Simple Cabibbo mixing of $b$ with $d, s$ would not occur because they have different spins, but mixing of the form $\mathrm{b}_{\mu}^{\prime}=c_{1} \mathrm{~b}_{\mu}+c_{2} \gamma_{\mu} \mathrm{d}+c_{3} \gamma_{\mu} \mathrm{s}+c_{4} \partial_{\mu} \mathrm{d}+c_{5} \partial_{\mu} \mathrm{s}$ can occur (and does in some supersymmetric theories with spontaneous symmetry breaking). This could lead to new approaches to calculations of quark mixing angles. There would be no difficulty in satisfying the constraint [16] that $\tau_{\mathrm{b}}<10^{-8}$ s. Decays could also occur via flavor changing neutral spin -0 or 2 mesons. The main constraints on these are the non-occurrence of $\tau \rightarrow$ eeē and $\mu \rightarrow$ eeē, which are important experiments. They can be satisfied by having no neutral currents with both flavor changing and flavor diagonal couplings, which will occur automatically in some theories. While we cannot give precise predictions, it will be important to check that $b$ decays have the expected patterns.

\footnotetext{
\#2 Less simplistically, there will presumably be a color group which is connected with binding forces, a flavor group to label different spin-1/2 states, and spin. Totally antisymmetric wavefunctions will then give the low lying states. The states $\tau, \mathrm{t}, \mathrm{b}, \nu_{\tau}$ will occur in a multiplet; the situation may be analogous to that with $\Delta, N$. It does not seem possible to decide between using three fundamental fermions

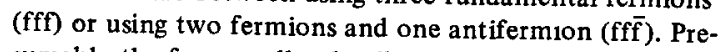
sumably the forces will only allow one to have low lying states. Perhaps the theories with a fermion and a meson can be reinterpreted with the meson as $\mathrm{f} \overline{\mathrm{f}}$, makıng some of the meson degrees of freedom dynamical ones in the $f \bar{f}$ system.
} 
Comments The interpretation of fermion masses is an important question. Why should the present fermions have $m \approx 0$ on the scale of a basic theory (where energy scales are of order of tens of $\mathrm{GeV}$ at least and could even be a Planck mass)? It could have happened that one could interpret the present fermions as having zero mass because they were coupled to a conserved supercurrent in the theory. Then some small breaking occurred for the charged states, which acquired a small mass. However, the experimental argument [15] against such a Goldstone fermion interpretation for the $v$ seems to apply here too, not changing even if the electron is also considered as massless. Theoretically also it does not seem possible to make a consistent scheme when the expected SU(2) X U(1) symmetry is included. Thus the fermion masses probably constitute a serious difficulty for theories of composite leptons and quarks. Of course, it may be possible to arrange a chiral invariance of some sort to account for small fermion masses, but while this assumption may be necessary to explain the small masses, we do not understand what its dynamical origin might be.

The existence of a 4 th generation (e.g. a new lepton) would considerably reduce the attraction of theories with lepton and quark substructure. Even then, however, the possibility that $\tau$ or $b$ have spin $-3 / 2$ should be carefully tested. Theories with spin-3/2 fermions also may have massive $s=0$ or 2 particles, so finding one of these could be relevant. Alternatively, of course, any discovery of deviations from pointlike behavior for leptons would dramatically establish the presence of substructure.

Because the theory with $\tau$, b having spin-3/2 might explain so much, it should be taken seriously and tested carefully, whatever the status of our theoretical understanding.

To conclude we want to emphasize that if the spins of $\tau$ and $b$ were found to be $3 / 2$ it would be of dramatic significance for ideas in particle physics. That leptons and quarks were made of more elementary objects would become a serious possibility, and the old $\mu / \mathrm{e}$ puzzle would conceivably be solved. Conversely, if $S_{\tau}$ $=S_{\mathrm{b}}=1 / 2$, it is likely that the solution to these prob. lems lies elsewhere, and in our view we can continue to hope that the present particles are the final ones to understand at the microscopic level.

We are grateful to Haim Harari for stımulating us to think about the subject matter of this paper, and to
G. Feldman, T. Sterlıng, R. Slansky, D. Freedman, J. LeVeılle, P. Fayet, C. Zachos, G. Farrar, and particularly M. Grisaru for help or discussions. One of us (G.L.K.) would like to thank the theory group at SLAC, where this work was begun, for their hospital1ty, and the Aspen Center for Physics for providing a stimulating atmosphere for discussions. This work was supported in part by the U.S. Department of Energy and in part by the National Science Foundation.

\section{References}

[1] J.C. Pat and A. Salam, Phys Lett 59B (1975) 265.

[2] O.W Greenberg and C Nelson, Phys. Rev D10 (1974) 2567.

[3] H. Terazawa, Y. Chikashige and K A Kama, Phys. Rev D15 (1977) 480, P G.O. Freund, Proc. 11 th Rencontre de Moriond (1976) Vol. 1, ed J. Tran Thanh Van

[4] Y. Ne'eman, Phys. Lett 82B (1979) 69.

[5] H. Harar1, Phys. Lett. 86B (1979) 83, this paper also has references to other unpublished work which we have not seen.

[6] Y.S. Tsa1, SLAC preprint SLAB-PUB-2105 (1978).

[7] See the talk of J Kirz, in: Proc. 19th Intern. Conf. on High energy physics (Tokyo, 1978), W. Bacino et al., Phys. Rev. Lett. 41 (1978) 13

[8] G. Feldman, Proc. 19th Intern. Conf. on High energy physıcs (Tokyo, 1978)

[9] W. Alles and V. Alles Borellı, Nuovo Cimento 35A (1976) 125;

Y Ahn, J. K1m and H.S. Song, Phys. Lett 73B (1978) 196;

W. Alles, Bologna preprint (June 1979).

[10] See the treatments of C. Llewellyn-Smith, Phys. Lett. 46B (1973) 233;

J. Cornwall, D Levin and G. Tiktopoulos, Phys. Rev D10 (1974) 1145

[11] For a review see T. Kinoshita, Proc 19th Intern. Conf on High energy physics (Tokyo, 1978); see S.J. Brodsky and G.P. LePage, Recent advances in bound state quantum electrodynamics, SLAC-PUB1966 , June 1977 for a possible lımit from $g-2$ on electron structure.

[12] For a review, see D Z. Freedman, Proc. 19th Intern Conf on High energy physics (Tokyo, 1978).

[13] G. Feldman, private communication

[14] C. Zachos, Extended supergravity with a gauged central charge, Ph.D. Thesis, Cal Tech (1979) Appendix B.

[15] W. Bardeen, unpublished; B. DeWitt and D Z. Freedman, Phys. Rev. Lett. 35 (1975) 827

[16] D. Cutts et al., Phys. Rev. Lett 41 (1978) 363, for constraints on neutral long-lived particles, see: H.R Gustafson et al, Phys. Rev. Lett. 37 (1976) 474 